\title{
Gelatin Nanoparticles Loaded with PMX-53 Prevents Alveolar Bone Loss in Miniature Swines Model of Periodontitis
}

\author{
Jiahui Pan ${ }^{1,2}$, Na Li 1,2, Qiuling Tang ${ }^{1,2}$, Gege Li $^{1,2}$, Yubo Hou ${ }^{1,2}$, Liuran Wang ${ }^{1,2}$, Weixian Yu ${ }^{1,2^{*}}$. \\ 1 Department of Periodontology, School and Hospital of Stomatology, Jilin University, Changchun 130021, \\ China; panjiahui0451@sina.com; 825900820@qq.com; 1099524983@qq.com; 1099075958@qq.com; \\ 1163586460@qq.com; 1164830162@qq.com; yu-wei-xian@163.com \\ 2 Jilin Provincial Key Laboratory of Tooth Development and Bone Remodeling, Changchun 130021, China \\ * Correspondence: yu-wei-xian@163.com; Tel.: +86-18943971853
}

\begin{abstract}
Background: The complement becomes a major link between infection and inflammatory pathology including periodontitis. Gingipians as important virulence factors of $P$. gingivalis have the activity of $\mathrm{C} 5$ convertase, could cleave $\mathrm{C} 5$ into fully functional $\mathrm{C} 5 \mathrm{a}$ to activate $\mathrm{C} 5 \mathrm{aR}$. The above process could be blocked by the C5aR antagonist (PMX-53) to suppress periodontal inflammation, and then achieves the purpose of treatment of periodontitis. Nanoparticles incorporated within gelatin are promising carrier system for drug delivery in recent years. This study aimed to investigate whether gelatin nanoparticles loaded with PMX-53 prevents alveolar bone resorption in miniature swines model of periodontitis; 2) Methods: Four miniature swines were placed ligatures for seven weeks to induce periodontitis. Then, animals were assigned randomly to four groups: minocycline-treated group, gelatin with PMX53-treated group, gelatin-treated group and a sham control group. They were treated with $1 \mathrm{ml}$ related drugs respectively. We showed that local treatments with gelatin nanoparticles loaded with PMX-53 could inhibit alveolar bone loss of periodontitis; 3) Results: Our study revealed that gelatin nanoparticles loaded with PMX-53 prevented alveolar bone resorption miniature swines model of periodontitis; and 4) Conclusions: We provided proof-of-concept for local targeting of gelatin nanoparticles loaded with PMX-53 as a powerful candidate for the treatment of periodontitis.
\end{abstract}

Keywords: periodontitis; PMX-53; miniature swines

\section{Introduction}

Periodontitis is a chronic inflammatory disease produced by gram-negative anaerobic bacteria predominantly which results in the destruction of the tooth' s supporting tissues and the alveolar bone resorption. It is well documented that periodontitis is initiated by oral pathogens embedded in gingival tissue to accumulate dental biofilms and related to a complex interplay of bacteria with host immune responses that eventually lead to progressive destruction of the periodontium[1]. The potential impact of many systemic disorders on the periodontium has been shown. Also, evidence suggests that periodontitis may be significantly associated with certain systemic diseases because of a translocation of periodontal bacteria into the systemic circulation such as cardiovascular disease, stroke, diabetes mellitus, ulcerative colitis, rheumatoid arthritis, preterm labor, and respiratory conditions[2,3]. Approximately $49 \%$ of the population suffers from generalized periodontitis [4]. Periodontal diseases coupled with systemic implications have a significant impact on the lives among the population. Hence, knowledge about prevention and treatment of periodontitis is urgently needed to improve the quality of life and maintain the health [5]. Therapy for periodontitis aims to reduce the pathogenic bacteria found in dental plaque to slow or arrest periodontal disease progression [6]. Conventional treatment methods are mechanical eradication of bacterial plaque included scaling and root planning (SPR), but the effectiveness of SPR is limited in inaccessible areas to periodontal instruments [7]. Local antimicrobial agents are used as an adjuvant factor in the 
clinical therapy [8]. Minocycline hydrochloride tetracycline broad-spectrum antibiotics are the most commonly used as local drug delivery agents [9]. However, the repeated and long-term use of antibiotics has potential side effects and the development of bacterial resistance, flora imbalance and superinfections has been reported [10,11]. Therefore, it is significant to develop a drug which has small side effects and independent intellectual property rights to treat periodontitis.

Porphyromonas gingivalis (P. gingivalis) is proposed to be a "keystone pathogen" causing microbial and immune dysbiosis in periodontitis. It has a number of virulence factors and extracellular proteases, such as lipopolysaccharide, gingipains, fimbriae, and capsule [12]. Gingipains are the kinds of cysteine proteinase including arginine-specific gingipains (Arg-gingipain-A, RgpA and Arg-gingipain-B, RgpB) and lysine-specific gingipain (Lys-gingipain, Kgp) [13]. The Arg-gingipains have C5 convertase activity which can degrade C5 into C5a to activate the C5a-C5aR signaling pathway to make periodontal immune inflammation [14]. Moreover, the C5aR crucially involved in immune subversion by P. gingivalis with TLR2 crosstalk [15]. PMX-53 is a polypeptide of the synthesis simulates chemical structure of $\mathrm{C} 5 \mathrm{aR}$ as a potent antagonist of $\mathrm{C} 5 \mathrm{aR}$ which could target to block the C5a-C5aR signaling pathway to develop anti-inflammatory effects [16]. Abe's studies have found that local administration of PMX-53 efficiently protected mice against the development of periodontitis by counteracting microbial immune evasion and suppressing the induction of proinflammatory cytokines in both preventive and therapeutic modes of treatment. In addition, PMX-53 also could reduce the destruction of the alveolar bone [17]. However, the duration of drug action is very short, so it needs to be injected frequently and multi-points injection is painful and inconvenient for the patients.

Gelatin, belonging to the family of the natural polymers derived from the hydrolysis of collagen, is a combination of high molecular weight water-soluble proteins [18]. It is inexpensive, biocompatible, biodegradable and low antigenic that considered a generally regarded as safe. Furthermore, due to their intrinsic protein structure could modify multiply coupling with crosslinkers and targeting-ligands which may be especially useful. It is already indispensably used in modern pharmaceutical and medical applications [19]. With the continuous development of biological materials science, nanotechnology has been widely concerned around the world. And the nanoparticles use, over this period, has been a particular focus [20]. Nanoparticles incorporated within gelatin are promising carrier system for drug delivery in recent years due to harmless, simple preparation process, strong target, improving the drug action concentration adequately, prolonging the drug action time, reducing the drug dosage greatly, and extending the intervals between patients visit [21,22] .

In this study, we showed that local administration of gelatin nanoparticles loaded with PMX-53 sustained release agents efficiently protected miniature swines against periodontal inflammation and bone loss in the treatment. The physiology, pathology, immunology, and anatomy structures of miniature swines are similar to those of humans. Our new findings therefore provide proof-of-concept for the efficacy of gelatin nanoparticles loaded with PMX-53 as a locally administered long-term therapeutic agent against periodontitis.

\section{Results}

\subsection{Establishing the miniature swines model of periodontitis}

Intraoral photographs showed the clinical assessments of the periodontal tissues of miniature swines. Before the experiment (-7 weeks), gingiva of each miniature swine was normal, healthy color without swelling, dental calculus. Probing the gingiva didn't bleed. Besides, neither periodontal pockets nor attachment loss were detected. The clinical periodontal index of each group was analyzed and there was no significant difference in all groups. Statistical analysis indicated periodontal conditions of each miniature swines were similar before the experiment. After establishing the periodontal models of miniature swines, immediately before treatment (week 0): the buccal gingiva of experimental teeth were swelling and a lot of obvious dental calculus were found around the teeth. Buccal gingiva bleeding with probing and the periodontal pockets easily were detected. The periodontium of experimental teeth was chronic inflammatory. There was no 
significant difference in all groups of clinical periodontal index. The results of X-ray images revealed that the alveolar bone of each miniature swine didn't resort obviously before modeling, while alveolar bone ridge of the experimental teeth reduced to the apical 1 / 2 significantly after modeling. There is no significant difference between the groups. Micro-CT and X-ray examinations showed similar results basically. There was an obvious decrease in the alveolar crest height after establishing miniature swines model of periodontitis. To sum up, establishing the periodontal model of miniature swines was successful.
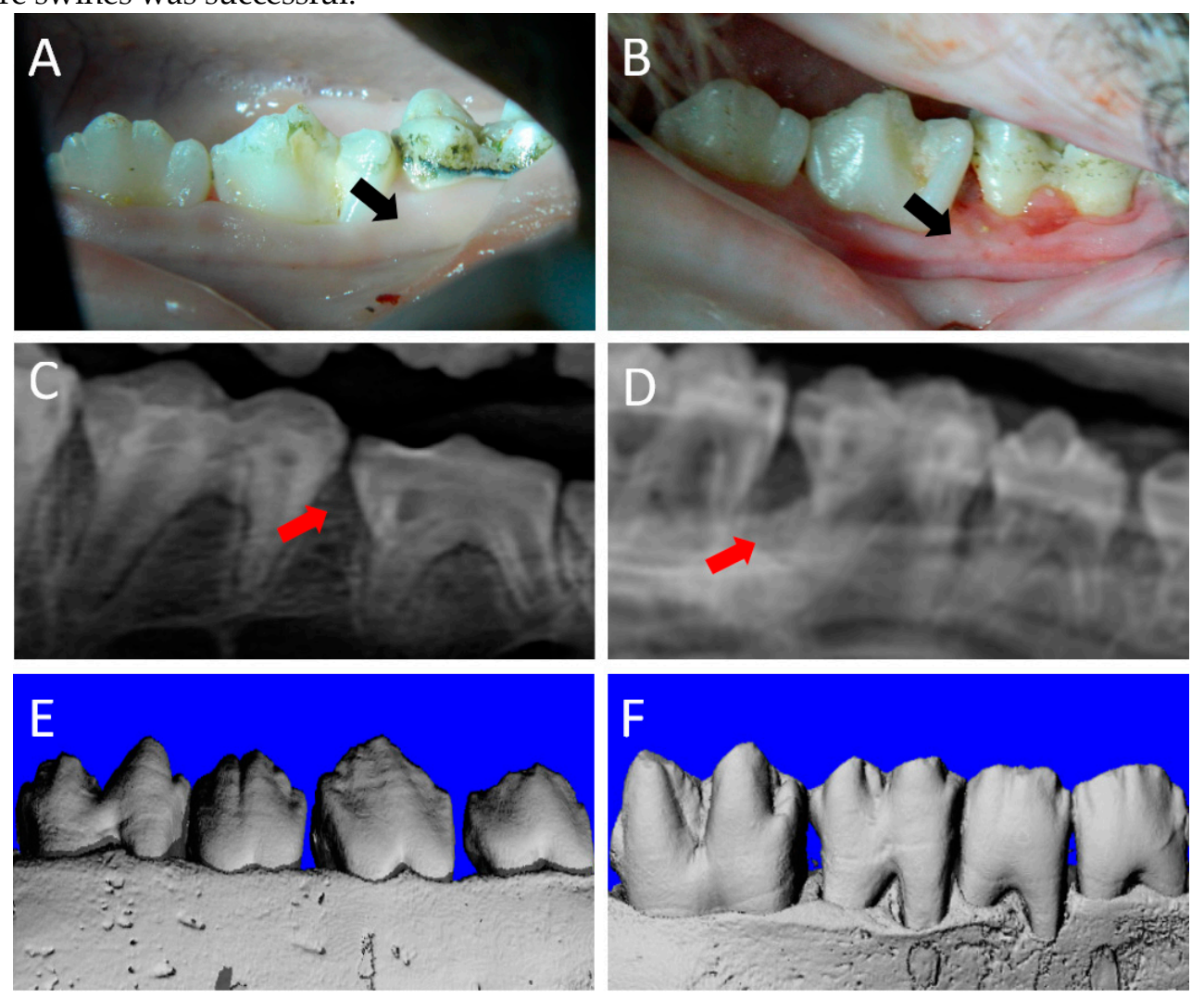

Figure 1. Establishing the miniature swines model of periodontitis. (A-C) Intraoral photographs of the periodontal tissues before modeling (-7 weeks) (A), ligation process (B) and after establishing the periodontal models of miniature swines (week 0) (C). The gingiva of the ligatured region and completed modeling were more swelling compared with healthy gingiva. $X$-ray images of alveolar bone before modeling (D) and completed modeling (E). Three-dimensional reconstruction of Mirco-CT of alveolar bone before modeling (F) and completed modeling (G). X-ray and Mirco-CT showed obvious alveolar bone loss of completed modeling compared with healthy miniature swines. The black arrowheads indicated the condition of gingiva in intraoral photographs. The red arrowheads indicated alveolar bone in X-ray images.

2.2 Intraoral photographs of periodontal tissue inflammation with treatment.

We generated periodontitis lesions in miniature swine, then conducted SPR and treated with minocycline, gelatin with PMX-53 or gelatin. After acquiring the intraoral photographs, animals were sacrificed at 4 weeks post treatment. We found that the periodontal condition had great improvement after 4 weeks treatment. Compared with untreatment, the periodontal tissue only conducted SPR reduced swelling a little, but the tissues were not restored to healthy levels (A). In contrast, periodontal tissue conducted SPR and treated with minocycline (B) and gelatin with PMX-53 (C) was restored to close to normal levels. The condition of treating with gelatin (D) was similar with only conducted SPR, but the gingival color had healthier changed. 

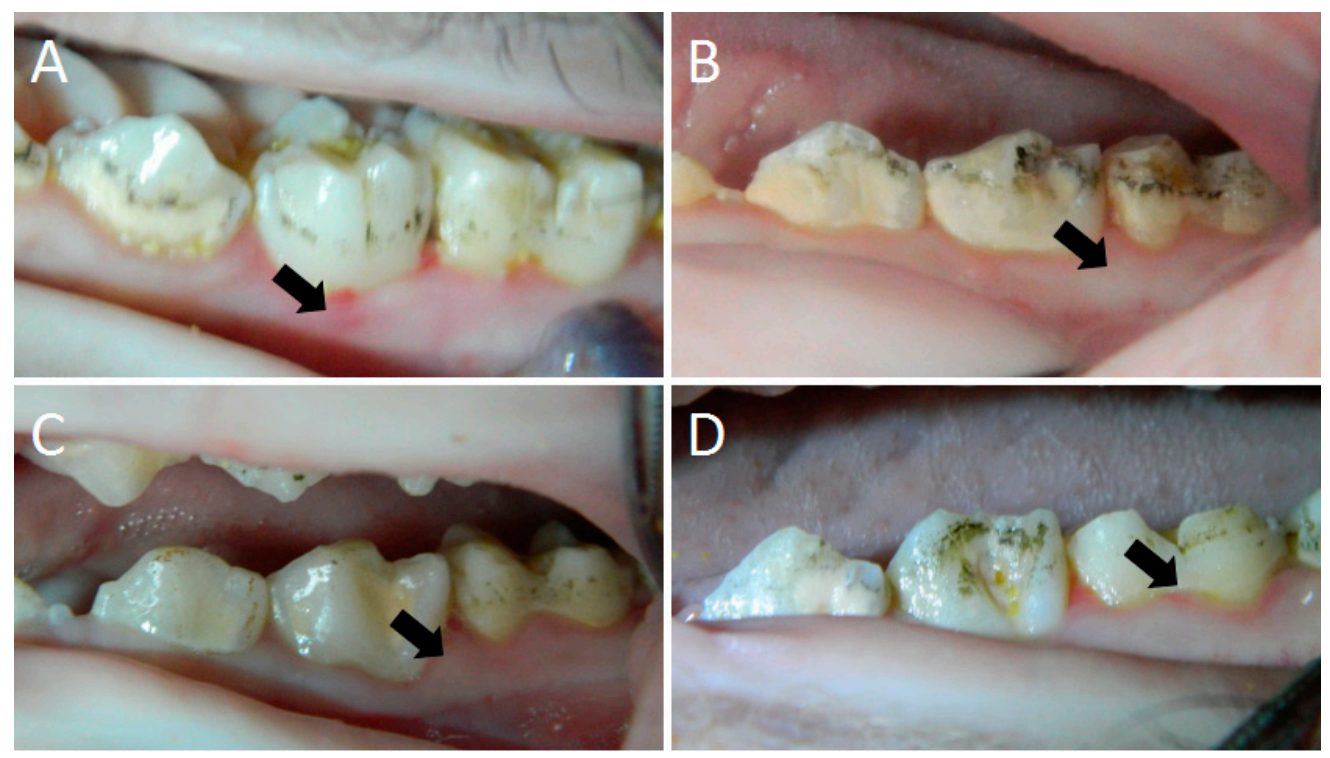

Figure 2. Intraoral photographs indicated periodontal tissue inflammation treated with gelatin nanoparticles loaded with PMX-53 in miniature pigs. Clinical assessments of the periodontal tissues treated 4 weeks with SPR in all of the 4 groups. In addition, each group treated with nothing more (A), minocycline (B), gelatin with PMX-53 (C), gelatin (D). The periodontal tissue only conducted SPR and adjuvant gelatin reduced swelling a little, but not restored to healthy levels. Periodontal tissue conducted SPR and treated with minocycline and gelatin with PMX-53 were restored to close to normal levels. The black arrowheads indicated the condition of gingiva in intraoral photographs.

2.3 Clinical assessments of the periodontal tissue inflammation with treatment photographs of periodontal tissue inflammation with treatment.

This time, values of the pocket depths (PD) (A) of 4 weeks were $3.58 \pm 0.51 \mathrm{~mm}$ in the sham control group, $2.71 \pm 0.75 \mathrm{~mm}$ in the minocycline-treated group, $2.54 \pm 0.66 \mathrm{~mm}$ in the gelatin with PMX53-treated group, and $3.50 \pm 0.72 \mathrm{~mm}$ in the gelatin-treated group. Statistical analysis manifested that among the minocycline-treated group, gelatin with PMX53-treated group and gelatin-treated group of 4 weeks increased the pocket depths with these groups of week 0 significantly. However, there was no significant difference between the sham control group of week 0 and 4 weeks. $\left({ }^{*} p<0.05\right.$, $\left.{ }^{* *} \mathrm{p}<0.01, \mathrm{n}=24\right)$ At week 0 , there was no significant difference of PD among the 4 groups. At 4 weeks post treatment, the PD were decreased apparently in the groups that received minocycline and gelatin with PMX-53 treatment with those in the sham control group (only SPR). Furthermore, there was significant difference between gelatin with PMX53-treated group and gelatin-treated group. $(\# \mathrm{p}<0.05, \# \mathrm{p}<0.01, \mathrm{n}=24)$. The sulcular bleeding index (SBI) (B) of 4 weeks values were $2.75 \pm 0.50$ in the sham control group, $2.25 \pm 0.46$ in the minocycline-treated group, $1.88 \pm 0.64$ in the gelatin with PMX53-treated group, and 2.50 \pm 0.53 in the gelatin-treated group. Statistical analysis indicated that the condition of SBI was much identical among the minocycline-treated group, gelatin with PMX53-treated group and gelatin-treated group of 4 weeks with these groups of week 0 . And after 4 weeks post treatment, the SBI were decreased significantly in the groups that received gelatin with PMX-53 treatment contrasted with the sham control group. $\left({ }^{*} \mathrm{p}<0.05, \mathrm{n}=8\right)$.

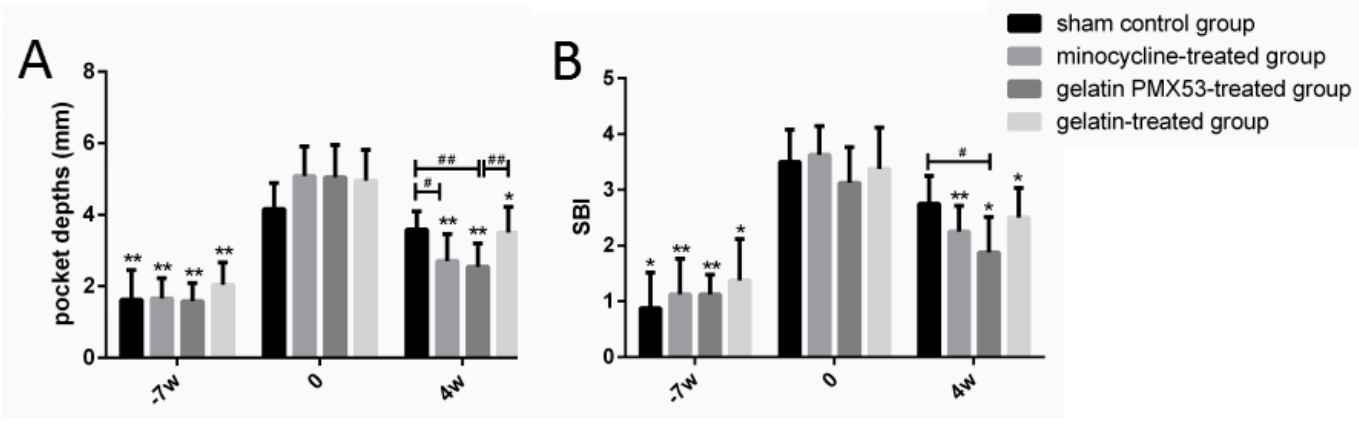


Figure 3. Clinical assessments of the periodontal tissue inflammation treated with gelatin nanoparticles loaded with PMX-53 in miniature pigs. (A) PD in the minocycline-treated group, gelatin with PMX53-treated group and gelatin-treated group of 4 weeks were less than these groups of week 0. ( ${ }^{*} \mathrm{p}<0.05,{ }^{* *} \mathrm{p}<0.01, \mathrm{n}=24$ ) Moreover, at 4 weeks post treatment, PD were less in the minocycline-treated group and gelatin with PMX53-treated group compared with the sham control group (\#p <0.05, \#\# <0.01, $\mathrm{n}=24$ ). (B) SBI in the minocycline-treated group, gelatin with PMX53-treated group and gelatin-treated group of 4 weeks were less than these groups of week 0. $\left({ }^{*} \mathrm{p}<0.05,{ }^{* *} \mathrm{p}<0.01, \mathrm{n}=8\right.$ ) Furthermore, at 4 weeks post treatment, the SBI were less in gelatin with PMX53-treated group compared with the sham control group $(\# \mathrm{p}<0.05$, \#\#p<0.01, $\mathrm{n}=24)$

\subsection{Three-dimensional reconstruction of Mirco-CT of aveolar bone}

The micro-computed tomography (Micro-CT) scanning and 3D reconstruction indicated that marked bone absorption of the buccal maxillary and mandibular fourth premolar and first molar on the both sides occurred in the sham control group (only SPR) (A). In the minocycline-treated (B) and gelatin with PMX53-treated group (C), alveolar bone resorption was considerably less than there was in the sham control group. In addition, the bone resorption in miniature swines treated with gelatin was a little lower than that in the sham control group.
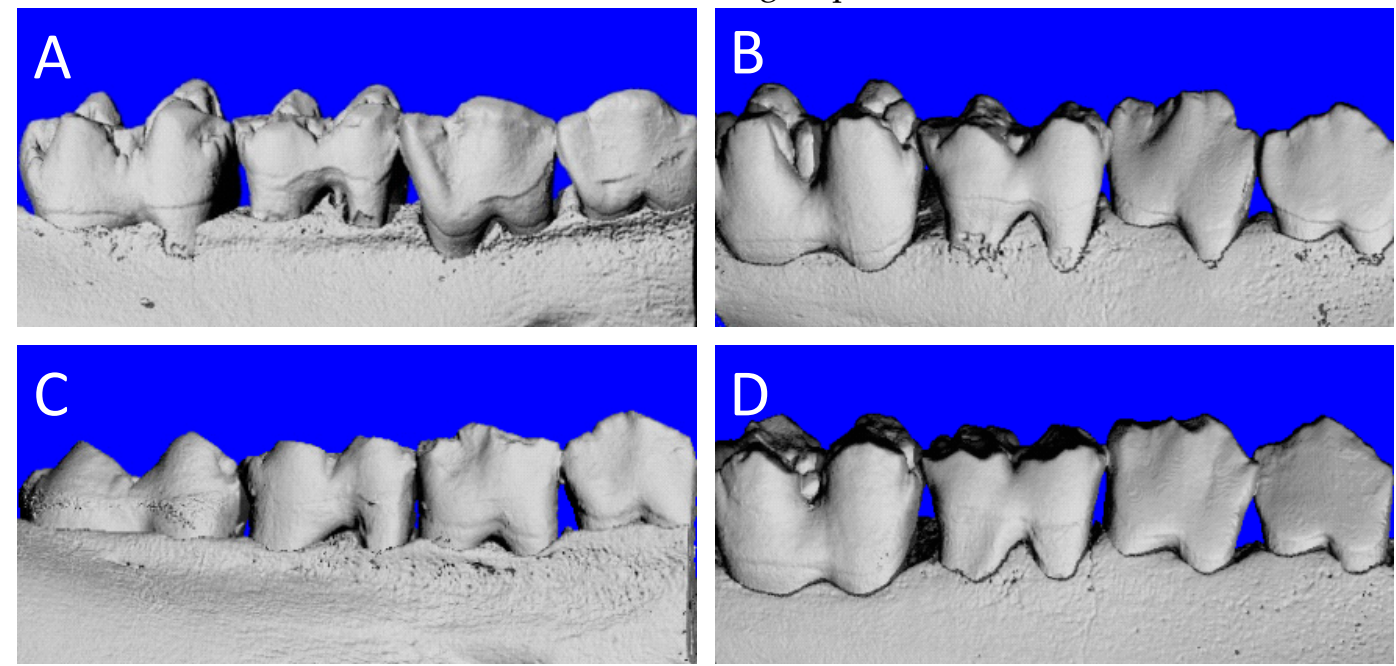

Figure 4. Gelatin nanoparticles loaded with PMX-53 prevented bone resorption. Three-dimensional reconstruction of Mirco-CT of treating with (A) SPR alone, (B) SPR and minocycline, (C) SPR and gelatin with PMX-53 and (D) SPR and gelatin provided visual references for changes induced. Micro-CT showed alveolar bone resorption was more obvious in the sham control group and gelatin-treated group than those in the minocycline-treated and gelatin with PMX53-treated group.

\subsection{The 3-dimensional region of interest for determination of bone volume changes}

The 3-dimensional region of interest for determination of bone volume changes was defined by a cuboidal bone body that vertically as the distance from the most coronal part of the alveolar bone crest $(\mathrm{ABC})$ to the maxillary and mandibular fourth premolar and first molar root apex. The length extended from the most mesial aspect of the maxillary and mandibular fourth premolar root to the most distal aspect of first molar root. Compared to miniature pig in the sham control group, receiving minocycline and gelatin with PMX-53 treatment showed higher bone volume/tissue volume (BV/TV), trabecular thickness (Tb.Th), and trabecular number (Th.N). It is obvious that the trabecular separation ( $\mathrm{Tb} . \mathrm{Sp}$ ) was lower in the gelatin with PMX53-treated group than that in the sham control group. The gelatin-treated group was associated with increasing tendency of BV/TV, $\mathrm{Tb}$.Th, Th.N, and decreased Tb.Sp compared to the sham control group, but only had the significant difference about $\mathrm{Tb}$.Th. There was no significant difference in BS/BV among the groups. 
A
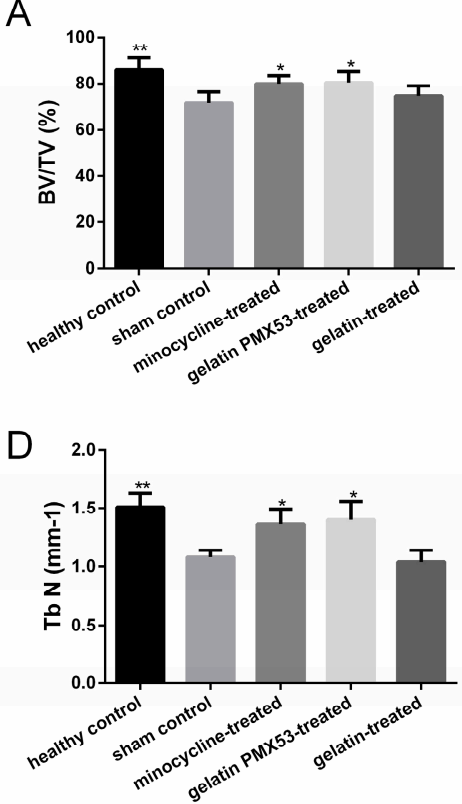

B
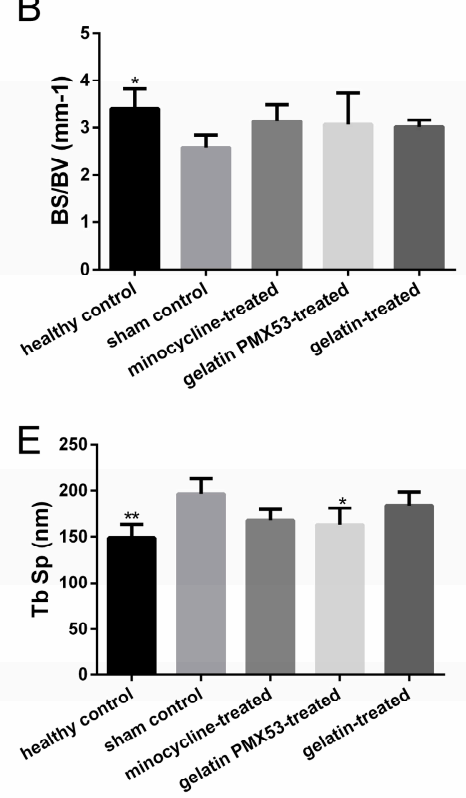

C

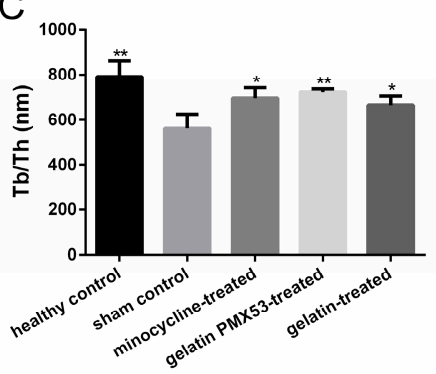

Figure 5. Effect of gelatin with PMX-53 by micro-CT quantitative analysis of the region of interest (ROI). (A-E) Analysis of Micro-CT volumetric parameters: bone volume/tissue volume (BV/TV), bone surface/bone volume (BS/BV), trabecular thickness (Tb.Th), trabecular number $(\mathrm{Th} . \mathrm{N})$, and trabecular separation (Tb.Sp). (All the data compared with the sham control group, ${ }^{*} \mathrm{p}<0.05,{ }^{* *}$ $\mathrm{p}<0.01, \mathrm{n}=4)$.

2.6 Measuring distance between the cement-enamel junction (CEJ) and the alveolar bone crest $(\mathrm{ABC})$

Alveolar bone loss was measured from the CEJ to the ABC at six points: mesiobuccal (MB), midbuccal, and distobuccal (DB) regions for maxillary and mandibular fourth premolar and first molar. Analysis of the CEJ-ABC linear distance after treatment. The values obtained for the six sites were summed to obtain the average values for alveolar bone resorption. The values of CEJ-ABC distance was $0.81 \pm 0.24 \mathrm{~mm}$ in the healthy control group, $3.29 \pm 0.44 \mathrm{~mm}$ in the sham control group, $2.16 \pm 0.41 \mathrm{~mm}$ in the minocycline-treated group, $1.94 \pm 0.70 \mathrm{~mm}$ in the gelatin PMX53-treated group, $2.90 \pm 0.76 \mathrm{~mm}$ in the gelatin-treated group. Statistical analysis indicated that the CEJ-ABC distance in both minocycline-treated group and gelatin with PMX53-treated group obviously lower than the sham control group. The CEJ-ABC distance in gelatin with PMX53-treated group was obviously lower contrast with gelatin-treated group. But there was no significant difference between sham control group and gelatin-treated group. $\left({ }^{*} \mathrm{p}<0.05,{ }^{* *} \mathrm{p}<0.01, \mathrm{n}=24\right)$ 


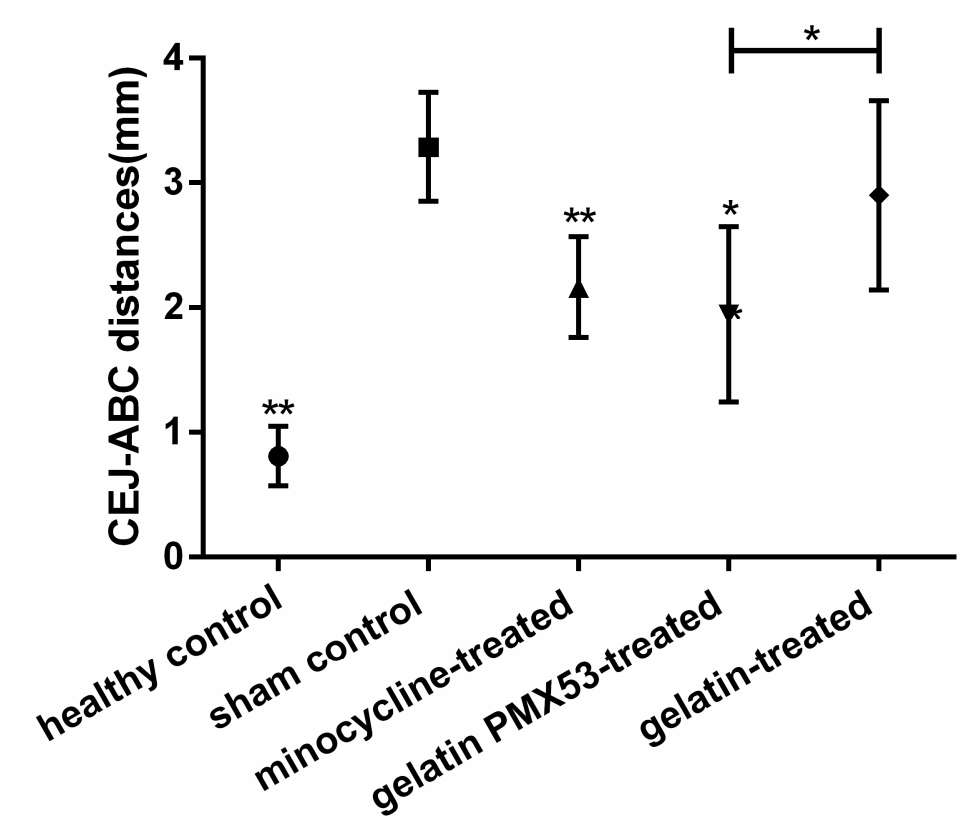

Figure 6. Distance between the cement-enamel junction (CEJ) and the alveolar bone crest (ABC) at distal, middle and mesial areas of vocal sides of the maxillary and mandibular fourth premolar and first molar in the five groups. ( ${ }^{*} \mathrm{p}<0.05,{ }^{* *} \mathrm{p}<0.01, \mathrm{n}=24$ ). The distance of CEJ-ABC both in the minocycline-treated group and gelatin with PMX53-treated group was obviously lower contrast with the sham control group. The results in the sham control group and gelatin-treated group had no significant difference.

\section{Discussion}

Porphyromonas gingivalis is a major causative agent of periodontitis, a chronic inflammatory disease of the supporting structures of the teeth. P. gingivalis produces a broad spectrum of virulence factors. Thereinto, gingipians play critical roles in the pathogenesis of periodontitis, more particularly in the complete system [23]. The gingipains have the activity of C5 convertase, could cleave $\mathrm{C} 5$ into fully functional $\mathrm{C} 5 \mathrm{a}$ to activate $\mathrm{C} 5 \mathrm{aR}$. The above process could be blocked by the $\mathrm{C} 5 \mathrm{aR}$ antagonist (PMX-53) to suppress local periodontal inflammation, and then achieves the purpose of treatment of periodontitis[24,25]. Related studies have shown that oral administration of PMX-53 can inhibit the occurrence and development of immune inflammation to treat rheumatoid arthritis [26]. In addition, it has been reported that local injected PMX-53 into periodontal tissue, could effectively inhibit periodontal tissue inflammation and alveolar bone resorption. Nanoparticles (NPs) have been developed rapidly over the past few years since they have shown great potential in drug delivery [27]. Gelatin Nanoparticles have attracted much attention because of high specific surface area, low particle size, non-toxic and non-antigenic properties. When the drug and gelatin were made into drug-loaded gelatin nanoparticles, the drug could be sustained released with the stable degradation of nanoparticles[28]. Taken together, these findings provide a theoretical foundation that gelatin nanoparticles loaded with PMX-53 are likely to be more effective to prevent periodontist in this study.

In this study, we established the model of periodontitis with silk suture in miniature swines. The oral maxillofacial region of miniature swines is similar to that of humans in anatomy, development, physiology, pathophysiology, and disease occurrence. So the miniature swines' model of periodontitis is superior to models using small animals. The results showed that typical periodontitis could be generated in miniature swines and that the general performances had features similar to those described in human cases of periodontitis. (Figure 1)

The present study showed that conduct SPR and local delivery minocycline ointment and gelatin nanoparticles loaded with PMX-53 could significantly inhibit gingival inflammation and alveolar bone resorption compared with conducting SPR alone or local delivery gelatin. Although 
minocycline and gelatin nanoparticles loaded with PMX-53 were less effective in the alveolar bone regeneration, could reduce the generation of osteoclast cytokines to avoid increasing alveolar bone resorption further $[29,30]$. The treated effects of minocycline and gelatin nanoparticles loaded with PMX-53 were essentially flat. Gelatin nanoparticles loaded with PMX-53 may have a slight advantage on the inflammatory effect, but there is no statistical significance, only the trend. It may be due to inadequate treatment time, the role of PMX-53 is not obvious to play. However the supreme advantage of gelatin nanoparticles loaded with PMX-53 was they could be used continuously. Minocycline therapy seems to be a less desirable choice because the development of bacterial resistance and flora imbalance will appear while using minocycline for a long time [31]. In the current study, we also found in the results of periodontal pocket depths and Tb.Th of micro-CT quantitative analysis, the periodontist was treated with gelatin had a certain degree of improvement compared to the sham control group. The results may be attributed to gelatin could provide hemostasis and facilitates cell adhesion and proliferation to promote wound healing Error! Reference source not found..This effect of trertment is relatively little and the amount of gelatin is not sufficient, therefore the treatment effect of gelatin is not shown in other results. In addition, the medication concentration in the periodontal pocket wasn't effective enough due to the limited penetration of the medication [33]. The medication in topical administration might lack sustenance with being squeezed out or diluted with crevicular fluid readily. These factors could make the discrepancies of experiments.

The authors did not examine the mechanism of gelatin nanoparticles loaded with PMX-53 treated on periodontitis. Hajishengallis's report has shown PMX-53 as C5aRA may exhibit anti-inflammatory action, against periodontitis. Firstly, it can block $\mathrm{C} 5 \mathrm{aR}$ access to P. gingivalis and thus deprive bacterium of a crucial immune subversion strategy. Secondly, C5aRA can abrogate the C5aR-TLR2 inflammatory synergism in the periodontal tissue [17].

In summary, our findings suggest that gelatin nanoparticles loaded with PMX-53 has a positive effect on miniature swines with experimentally induced periodontitis. They arrest the development of periodontitis by counteracting inflammation and bone resorption. Future studies should conduct histological assessment and Immunohistochemistry for periodontal tissue of miniature swines. Taken together, our study strongly suggests that locally applied gelatin nanoparticles loaded with PMX-53 may potentially find application for the treatment protocol of periodontitis.

\section{Materials and Methods}

\subsection{Bacteria}

Cultures of Porphyromonas gingivalis (standard-type strain ATCC33277, Capital Medical University School presented, Beijing, China) were inoculated into on blood agar plates supplemented with hemin ( $5 \mu \mathrm{g} / \mathrm{ml}$, Sigma 51280, Sigma, St Louis, MO, USA) in an anaerobic chamber flushed with N2 $(80 \%), \mathrm{CO} 2(10 \%)$ and $\mathrm{H} 2(10 \%)$ at $37^{\circ} \mathrm{C}$ for $72 \mathrm{~h}$. Cultured P. gingivalis was diluted to $1.0^{*} 109$ colony-forming units with sterile phosphate-buffered saline for oral infection.

\subsection{Preparation of Gelatin Nanoparticles Loaded with PMX-53}

All reagents used in this study were commercially available. $500 \mathrm{mg}$ gelatin (Sigma, St Louis, $\mathrm{MO}$, USA) was dissolved in $10 \mathrm{ml}$ de-ionized water at $40^{\circ} \mathrm{C}$. Then, the solution was added into $10 \mathrm{mg}$ PMX-53 (GL Biochem Ltd, Shanghai, China) to produce a colloidal dispersion. Afterwards, $500 \mu \mathrm{l}$ gluteraldehyde was added into the dispersion to initiate the cross-linking reaction formed nanoparticles and to prevent their swelling. Magnetic stirring for 3-5 minutes was performed. Consequently, the colloidal dispersion volume was dissolved in $25 \mathrm{ml}$ using deionized water. Finally, the prepared nanospheres were separated by centrifugation at 13,000 rpm for 25 minutes to gain gelatin nanoparticles loaded with PMX-53 powder.

\subsection{Animals}

The study was approved by the Animal Ethics Committee of Jilin University. The animal protocol for this study was approved by the Animal Care and Experiment. Four male Bama suckling 
miniature swines, 12 months old and weighing $40 \mathrm{~kg}$, were purchased from Taizhou experimental miniature swines breeding base (Taizhou, China). The miniature swines lived in the animal experimental base of Heping campus of Jilin University to adapt the environment for 7 days before establishing the model of periodontitis.

\subsection{Experimental periodontitis model}

Four miniature swines were used to generate periodontitis lesions. Among them, one miniature swine only established the model of periodontitis on the right side, in order to retain the left side as the healthy control. All surgical procedures were performed under general anesthesia, achieved with Xylazine Hydrochloride injection $(0.2 \mathrm{mg} / \mathrm{kg}$ intramuscularly [IM]) and Midazolam $(0.05 \mathrm{mg} / \mathrm{kg}$ IM) for maintenance. Local anesthesia (2\% lidocaine with 1: 200,000 epinephrine) was administered to the gingiva of maxillary and mandibular forth premolar and first premolar on both sides for anesthesia and hemostasis. Ligature of 10th surgical silk suture (Xihubiom, Hangzhou, China) was placed around the maxillary and mandibular fourth premolar and first molar on both sides to induce periodontitis. After ligature placement, $5 \mathrm{ml}$ cultures of $\mathrm{P}$. gingivalis were inoculated into the gingival crevice of the experimental teeth for 7 weeks, once a week. During this time, the miniature swines were fed with regular food and water. The ligatures were removed after 7 weeks. All animals were housed in a temperature $\left(24^{\circ} \mathrm{C}\right)$ - and humidity-controlled room with a 12-h light/12-h dark cycle. During the ligature period, the miniature swines were checked once a week to ensure the success of the experimentally induced periodontitis.

\subsection{Treatments}

The left side alveolar of mininature swine which was't established model was served as the healthy control group, and the rest right side as the sham control group. Other three miniature swines were randomly assigned to one of three groups: a minocycline-treated group, a gelatin with PMX53-treated group and a gelatin-treated group. After establishing the model of periodontitis, each swines was conducted the scaling and root planing (SPR) of experimental teeth. The sham control group wasn't treated with local medicine, and the miniature swines in other groups were treated with $1 \mathrm{ml}$ minocycline, gelatin with PMX-53, and gelatin alone, respectively, into the gingival sulcus for 4 times in one-week intervals. Because gelatin nanoparticles loaded with PMX-53 is powder, so this material was suspensed with $3 \%$ sodium carboxymethyl cellulose to make the soliquoid.

\subsection{Clinical assessments}

Animals were monitored weekly to provide assess healing and inflammation. Clinical assessments, including pocket depth (PD) and sulcus bleeding index (SBI) were performed all experimental teeth each week. After 4 weeks treatment, all animals were sacrificed, and the samples from the experimental area were harvested and fixed with $4 \%$ paraformaldehyde for 48 hours.

\subsection{X-ray and Micro-computed tomography analysis}

Alveolar bone resorption was examined by X-ray (Sirona, Bensheim, Germany) after completing the model of periodontitis at week 0 . After treating the periodontitis of miniature swines, the fixed alveolar bone included four teeth which were two experimental teeth and two adjacent teeth on both sides were scanned with a micro-computed tomography (Micro-CT) scanner (Micro-CT50 Scanco Medical, Basserdorf, Switzerland). The system was set to $50 \mathrm{KV}$, with a current of $500 \mu \mathrm{A}$ and a fixed exposure time of $900 \mathrm{~ms}$. Images were obtained with a resolution of $2048^{*} 2048$ pixels and a voxel size of $10^{*} 10^{*} 10 \mu \mathrm{m} 3$. The three-dimensional images were reconstructed using IPL image-processing software (Scanco Medical, Bassersdorf, Switzerland) for quantitative and qualitative evaluation by computer (threshold $=200$ ). The 3-dimensional region of interest $(\mathrm{ROI})$ for determination of bone volume changes was defined a cuboidal bone body that vertically as the distance from the most coronal part of the alveolar bone crest (ABC) to the maxillary and mandibular fourth premolar and first molar root apex. The length extended from the most mesial 
aspect of the maxillary and mandibular fourth premolar root to the most distal aspect of first molar root. The following parameters of micro-architecture were assessed in the ROI images: bone volume/tissue volume (BV/TV), bone surface/bone volume (BS/BV), trabecular thickness (Tb.Th), trabecular number (Tb.N), and trabecular separation (Tb.Sp). The BV/TV value indicates the portion of mineralized tissue; $\mathrm{Tb}$.Th, $\mathrm{Tb} . \mathrm{Sp}$ and $\mathrm{Tb} . \mathrm{N}$ provide detailed information on the amount, thickness and organization of trabecular bone.

Alveolar bone loss (ABL), represented by the linear distance from the cemento-enamel junction (CEJ)to the alveolar bone crest (ABC) of the mesial, middle, and distal sites on buccal sides (three sites each tooth) of experimental teeth was measured on the computed tomography imaging indicated the severity of periodontitis during the clinical examinations, and was measured in millimeters. The average values of the 24 sites were calculated as alveolar bone resorption of one miniature swine. Additionally, a high ABL value meant heavy bone loss, whereas a low ABL value indicated a healthier alveolar bone.

\subsection{Statistical analysis}

All statistical values were performed with a statistical package for the social sciences (SPSS) 17.0 statistical software (SPSS Inc, Chicago, IL, USA). Quantitative data were expressed as the mean \pm standard deviation (SD). Statistical significance was determined by the independent sample test or One-way ANOVA analysis of variance. A value of $p<0.05$ was considered statistically significant.Materials and Methods should be described with sufficient details to allow others to replicate and build on published results. Please note that publication of your manuscript implicates that you must make all materials, data, computer code, and protocols associated with the publication available to readers. Please disclose at the submission stage any restrictions on the availability of materials or information. New methods and protocols should be described in detail while well-established methods can be briefly described and appropriately cited.

\section{Conclusions}

The present study demonstrates the effect of using gelatin nanoparticles loaded with PMX-53 as a C5aR antagonist to treat periodontitis in a miniature swine preclinical model.

Acknowledgments: This research was supported by the National Natural Science Foundation of Jilin Province (No: 20150101076JC)

Author Contributions: Jiahui Pan and Na Li conceived and designed the experiments; Jiahui Pan, $\mathrm{Na} \mathrm{Li}$, Qiuling Tang, Gege Li and Yubo Hou performed the experiments; Jiahui Pan and QiulingTang analyzed the data; Liuran Wang and WeixianYu contributed reagents/materials/analysis tools; Jiahui Pan wrote and reviewed the manuscript; All authors read and approved the final manuscript.

Conflicts of Interest: The authors declare no conflict of interest. 


\section{References}

1. Horie, M.; Yamaguchi, Y.; Saito, A.; Nagase, T.; Lizio, M.; Itoh, M.; Kawaji, H.; Lassmann, T.; Carninci, P.; Forrest, A.R., et al. Transcriptome analysis of periodontitis-associated fibroblasts by cage sequencing identified dlx5 and runx2 long variant as novel regulators involved in periodontitis. Scientific reports 2016, 6, 33666.

2. Khatri, R.; Deshmukh, J.; Shrivastava, R.; Gupta, S.; Kawadkar, A.; Vinaya Kumar, K. Is periodontitis an independent risk factor for subclinical atherosclerosis? Singapore dental journal 2016, 37, 9-13.

3. Ebersole, J.L.; Holt, S.C.; Cappelli, D. Periodontitis in pregnant baboons: Systemic inflammation and adaptive immune responses and pregnancy outcomes in a baboon model. Journal of periodontal research 2014, 49, 226-236.

4. Lundmark, A.; Davanian, H.; Bage, T.; Johannsen, G.; Koro, C.; Lundeberg, J.; Yucel-Lindberg, T. Transcriptome analysis reveals mucin 4 to be highly associated with periodontitis and identifies pleckstrin as a link to systemic diseases. Scientific reports 2015, 5, 18475.

5. Bai, Y.; Wei, Y.; Wu, L.; Wei, J.; Wang, X.; Bai, Y. C/ebp beta mediates endoplasmic reticulum stress regulated inflammatory response and extracellular matrix degradation in lps-stimulated human periodontal ligament cells. International journal of molecular sciences 2016, 17, 385.

6. Hasturk, H.; Kantarci, A.; Ebrahimi, N.; Andry, C.; Holick, M.; Jones, V.L.; Van Dyke, T.E. Topical h2 antagonist prevents periodontitis in a rabbit model. Infection and immunity 2006, 74, 2402-2414.

7. Nagasri, M.; Madhulatha, M.; Musalaiah, S.V.; Kumar, P.A.; Krishna, C.H.; Kumar, P.M. Efficacy of curcumin as an adjunct to scaling and root planning in chronic periodontitis patients: A clinical and microbiological study. Journal of pharmacy \& bioallied sciences 2015, 7, S554-558.

8. Sinha, S.; Kumar, S.; Dagli, N.; Dagli, R.J. Effect of tetracycline hcl in the treatment of chronic periodontitis - a clinical study. Journal of International Society of Preventive \& Community Dentistry 2014, 4, 149-153.

9. Sadaf, N.; Anoop, B.; Dakshina, B.; Shweta, B. Evaluation of efficacy of tetracycline fibers in conjunction with scaling and root planing in patients with chronic periodontitis. Journal of Indian Society of Periodontology 2012, 16, 392-397.

10. Lee, B.S.; Lee, C.C.; Wang, Y.P.; Chen, H.J.; Lai, C.H.; Hsieh, W.L.; Chen, Y.W. Controlled-release of tetracycline and lovastatin by poly(d,l-lactide-co-glycolide acid)-chitosan nanoparticles enhances periodontal regeneration in dogs. International journal of nanomedicine 2016, 11, 285-297.

11. Perayil, J.; Menon, K.S.; Biswas, R.; Fenol, A.; Vyloppillil, R. Comparison of the efficacy of subgingival irrigation with $2 \%$ povidone-iodine and tetracycline hcl in subjects with chronic moderate periodontitis: A clinico microbiological study. Dental research journal 2016, 13, 98-109. 
12. Baek, K.J.; Ji, S.; Kim, Y.C.; Choi, Y. Association of the invasion ability of porphyromonas gingivalis with the severity of periodontitis. Virulence 2015, 6, 274-281.

13. Jayaprakash, K.; Khalaf, H.; Bengtsson, T. Gingipains from porphyromonas gingivalis play a significant role in induction and regulation of cxcl8 in thp-1 cells. BMC microbiology 2014, 14, 193.

14. Popadiak, K.; Potempa, J.; Riesbeck, K.; Blom, A.M. Biphasic effect of gingipains from porphyromonas gingivalis on the human complement system. Journal of immunology 2007, $178,7242-7250$.

15. Hajishengallis, G.; Lamont, R.J. Beyond the red complex and into more complexity: The polymicrobial synergy and dysbiosis (psd) model of periodontal disease etiology. Molecular oral microbiology 2012, 27, 409-419.

16. Tamamis, P.; Kieslich, C.A.; Nikiforovich, G.V.; Woodruff, T.M.; Morikis, D.; Archontis, G. Insights into the mechanism of c5ar inhibition by pmx53 via implicit solvent molecular dynamics simulations and docking. BMC biophysics 2014, 7, 5 .

17. Abe, T.; Hosur, K.B.; Hajishengallis, E.; Reis, E.S.; Ricklin, D.; Lambris, J.D.; Hajishengallis, G. Local complement-targeted intervention in periodontitis: Proof-of-concept using a c5a receptor (cd88) antagonist. Journal of immunology 2012, 189, 5442-5448.

18. Zhuang, H.; Bu, S.; Hua, L.; Darabi, M.A.; Cao, X.; Xing, M. Gelatin-methacrylamide gel loaded with microspheres to deliver gdnf in bilayer collagen conduit promoting sciatic nerve growth. International journal of nanomedicine 2016, 11, 1383-1394.

19. Han, S.; Li, M.; Liu, X.; Gao, H.; Wu, Y. Construction of amphiphilic copolymer nanoparticles based on gelatin as drug carriers for doxorubicin delivery. Colloids and surfaces. B, Biointerfaces 2013, 102, 833-841.

20. Sapra, M.; Pawar, A.A.; Venkataraman, C. A single-step aerosol process for in-situ surface modification of nanoparticles: Preparation of stable aqueous nanoparticle suspensions. Journal of colloid and interface science 2016, 464, 167-174.

21. Singh, A.; Xu, J.; Mattheolabakis, G.; Amiji, M. Egfr-targeted gelatin nanoparticles for systemic administration of gemcitabine in an orthotopic pancreatic cancer model. Nanomedicine : nanotechnology, biology, and medicine 2016, 12, 589-600.

22. Sahoo, N.; Sahoo, R.K.; Biswas, N.; Guha, A.; Kuotsu, K. Recent advancement of gelatin nanoparticles in drug and vaccine delivery. International journal of biological macromolecules 2015, 81, 317-331.

23. Koro, C.; Bielecka, E.; Dahl-Knudsen, A.; Enghild, J.J.; Scavenius, C.; Brun, J.G.; Binder, V.; Hellvard, A.; Bergum, B.; Jonsson, R., et al. Carbamylation of immunoglobulin abrogates activation of the classical complement pathway. European journal of immunology 2014, 44, 3403-3412.

24. Tamamis, P.; Kieslich, C.A.; Nikiforovich, G.V.; Woodruff, T.M.; Morikis, D.; Archontis, G. Insights into the mechanism of c5ar inhibition by pmx53 via implicit solvent molecular dynamics simulations and docking. BMC biophysics 2014, 7, 5.

25. Iyer, A.; Woodruff, T.M.; Wu, M.C.; Stylianou, C.; Reid, R.C.; Fairlie, D.P.; Taylor, S.M.; Brown, L. Inhibition of inflammation and fibrosis by a complement c5a receptor antagonist in doca-salt hypertensive rats. Journal of cardiovascular pharmacology 2011, 58, 479-486. 
26. Vergunst, C.E.; Gerlag, D.M.; Dinant, H.; Schulz, L.; Vinkenoog, M.; Smeets, T.J.; Sanders, M.E.; Reedquist, K.A.; Tak, P.P. Blocking the receptor for c5a in patients with rheumatoid arthritis does not reduce synovial inflammation. Rheumatology 2007, 46, 1773-1778.

27. Yao, W.; Xu, P.; Pang, Z.; Zhao, J.; Chai, Z.; Li, X.; Li, H.; Jiang, M.; Cheng, H.; Zhang, B., et al . Local delivery of minocycline-loaded peg-pla nanoparticles for the enhanced treatment of periodontitis in dogs. International journal of nanomedicine 2014, 9, 3963-3970.

28. Patra, S.; Basak, P.; Tibarewala, D.N. Synthesis of gelatin nano/submicron particles by binary nonsolvent aided coacervation (bnac) method. Materials science E engineering. C, Materials for biological applications 2016, 59, 310-318.

29. Nagasawa, T.; Arai, M.; Togari, A. Inhibitory effect of minocycline on osteoclastogenesis in mouse bone marrow cells. Archives of oral biology 2011, 56, 924-931.

30. Niwa, T.; Mizukoshi, K.; Azuma, Y.; Kashimata, M.; Shibutani, T. Fundamental study of osteoclast chemotaxis toward chemoattractants expressed in periodontitis. Journal of periodontal research 2013, 48, 773-780.

31. Cheng, Q.; Gao, W.; Cao, B.; Liu, Y.; Zeng, Z.; Wang, Z. [comparison of the effects of ginkgo biloba extract and minocycline hydrochlovide on periodontitis]. Zhonghua kou qiang yi xue za zhi = Zhonghua kouqiang yixue zazhi = Chinese journal of stomatology 2014, 49, 347-351.

32. Boateng, J.; Burgos-Amador, R.; Okeke, O.; Pawar, H. Composite alginate and gelatin based bio-polymeric wafers containing silver sulfadiazine for wound healing. International journal of biological macromolecules 2015, 79, 63-71.

33. Xu, Y.; Wei, W. A comparative study of systemic subantimicrobial and topical treatment of minocycline in experimental periodontitis of rats. Archives of oral biology 2006, 51, 794-803.

(C) 2017 by the authors; licensee Preprints, Basel, Switzerland. This article is an open access article distributed under the terms and conditions of the Creative Commons by Attribution (CC-BY) license (http://creativecommons.org/licenses/by/4.0/). 